\title{
Resonant electron transmission through a finite quantum spin chain
}

\author{
Y. Avishai ${ }^{1,2}$ and Y. Tokura ${ }^{1}$ \\ 1 NTT Basic Research Laboratory, 3-1 Morinosato, Wakamiya, 243-01 Japan \\ 2 Ilze Katz Center and Department of Physics, Ben-Gurion University of the Negev, Beersheva, Israel
}

\begin{abstract}
Electron transport in a finite one dimensional quantum spin chain (with ferromagnetic exchange) is studied within an $s-d$ exchange Hamiltonian. Spin transfer coefficients strongly depend on the sign of the $s-d$ exchange constant. For a ferromagnetic coupling, they exhibit a novel resonant pattern, reflecting the salient features of the combined electron-spin system. Spin-flip processes are inelastic and feasible at finite voltage or at finite temperature.
\end{abstract}

Motivation and scope: There is a growing interest in electronic devices which transport electron spin [1] (together with its charge). Beside the Kondo physics [2], electron transmission through a region containing a lattice of magnetic atoms (mainly a domain wall) is a focus of experimental [3] [4] [5] [6] and theoretical [7] [8] [9] investigations. In this context, the basic ingredient is an exchange interaction $g \mathbf{s} \cdot \mathbf{S}_{n}$ between the electron spin operator $\mathbf{s}$ and an atomic spin $\mathbf{S}_{n}$ localized at a point $x_{n}$, with an "s-d" coupling $g$. Most theoretical works treat the atomic spins either purely classically (as localized magnetic fields), or semi classically (the operator $\mathbf{S}_{n}$ is replaced by its expectation value $\left\langle\mathbf{S}_{n}\right\rangle$ in the ground state of the quantum spin system). It is expected, however, that the quantum nature of the magnetic system will be crucial at low temperatures.

In the present work, transmission of electrons through a finite one dimensional quantum spin $1 / 2$ chain (with ferromagnetic exchange) is studied, starting from an $s-d$ exchange Hamiltonian. The many body Kondo problem is avoided by an application of a magnetic field which removes the degeneracy of the ground state. It also enables cutting the spectrum of the spin system off at the one magnon level. The ensuing scattering formalism can then be handled within a transfer matrix algorithm, in terms of which the spin transfer coefficients (STC) are calculated as function of the electron Fermi energy. It is found that for ferromagnetic $s-d$ exchange, the STC display a novel resonant structure which manifests the richness of the combined electron spin chain system.

Hamiltonian: Consider electrons (mass $m$ and charge $-e$ ) moving in a one dimensional wire (along $x$ ) interacting with a chain of quantum spins (e.g. magnetic atoms) $\mathbf{S}_{n},\left(n=1,2, \ldots N, \mathbf{S}_{N+1}=\mathbf{S}_{1}\right)$ localized at points $x_{n}=n a(n=1,2, \ldots N)$, subject to a perpendicular magnetic field $\mathbf{H}=H_{z} \hat{\mathbf{z}}$. Henceforth $a$ and $\hbar^{2} / 2 m a^{2}$ are exploited such that all length and energy quantities are dimensionless. The Hamiltonian of the system $H=H_{e}+H_{s-d}+H_{S}$ contains an electronic part $H_{e}$, an electron-spin $s-d$ exchange interaction $H_{s-d}$ and a term $H_{S}$ controlling the isolated spin system. The latter is,

$$
H_{S}=-\sum_{n}\left(J \mathbf{S}_{n} \cdot \mathbf{S}_{n+1}+E_{Z} S_{n z}\right),
$$

where $J>0$ and $E_{Z}$ is the atomic Zeeman energy.
The spin Hamiltonian $H_{S}$ is projected on the subspace spanned by its $N+1$ lowest energy states $\mid k>(k=$ $0,1, \ldots N)$. If the magnetic field is strong enough such that $E_{Z}>J S$ then, beside the (non degenerate) ground state $\mid k=0>$ (with all spins up along $\hat{\mathbf{z}}$ and energy $E_{0}=-N\left(S^{2} J+E_{Z} S\right)$ and $\left.\sum_{n} S_{n z}=\frac{N}{2}\right)$, these are the one magnon spin waves (OMSW) with $\sum_{n} S_{n z}=\frac{N}{2}-1$. In terms of states $\left|n>\equiv S_{n}^{-}\right| 0>$, the OMSW and their corresponding energies are,

$$
\begin{aligned}
& \left|k>=\frac{1}{\sqrt{N}} \sum_{n=1}^{N} e^{i \frac{2 \pi}{N} k n}\right| n>, \quad k=1,2, \ldots N \\
& E_{k}=E_{0}+4 S J \sin ^{2} \frac{\pi k}{N}+2 S E_{Z} .
\end{aligned}
$$

Cutting the spectrum off at the OMSW states level is justified since above it there is a gap of $2 S E_{Z}$ until the lowest energy of two magnon spin waves.

The electronic and $s-d$ parts of the Hamiltonian should, in principle, manifest the many-body aspects of the problem (in the Kondo sense). In first quantization that amounts to, $H_{e}=-\sum_{i} \frac{d^{2}}{d x_{i}^{2}}-h_{e} \sum_{i} s_{i z}$ and an exchange part $H_{s-d}=g \sum_{i} \sum_{n} \delta\left(x_{i}-n\right) \mathbf{s}_{i} \cdot \mathbf{S}_{n}\left(h_{e}\right.$ is the electronic Zeeman energy). The sum over $i$ runs, principally, on all the electrons in the wire. However, due to the presence of an external magnetic field, the ground state of the spin system is non degenerate and the Kondo effect is absent. Hence, the dynamics of the system is adequately described by the single electron $s-d$ exchange Hamiltonian,

$$
H=-\frac{d^{2}}{d x^{2}}-h_{e} s_{z}+g \sum_{n} \delta(x-n) \mathbf{s} \cdot \mathbf{S}_{n}+H_{S} .
$$

The Schrödinger equation is then,

$$
H \Psi\left(x ;\left\{S_{n}\right\}\right)=E \Psi\left(x ;\left\{S_{n}\right\}\right),
$$

where $\Psi\left(x ;\left\{S_{n}\right\}\right)$ and $E$ are the total wave function and total energy (electron and spin system).

The scattering problem: Between spins and outside the spin system, electrons propagate as plane waves with momenta $\left.p_{\sigma k}=\sqrt{(} E-E_{k}+\sigma h_{e}\right)$, (some of which might be purely imaginary), where $\sigma= \pm \frac{1}{2}$, (alternatively 
$\sigma=\uparrow \downarrow)$ is the electron spin projection along $\hat{\mathbf{z}}$. Solution of the scattering problem means the evaluation of transmission amplitudes $t_{\sigma^{\prime} k^{\prime} \sigma k}(E)$, in which $\sigma k$ are initial electron and spin system quantum numbers, while $\sigma^{\prime} k^{\prime}$ are the final ones. To extract the transmission amplitudes from the Schrödinger equation, note that the presence of spins at isolated points suggests using a transfer matrix formalism. For $x_{n-1}<x<x_{n}$ the total wave function is expanded in electron spinors $\chi_{\sigma}$ and spin states $\mid k>$,

$$
\begin{gathered}
\Psi\left(x ;\left\{S_{n}\right\}\right)=\sum_{\sigma^{\prime} k^{\prime}} \psi_{n \sigma^{\prime} k^{\prime}}(x) \chi_{\sigma^{\prime}} \otimes \mid k^{\prime}> \\
\psi_{n \sigma^{\prime} k^{\prime}}(x)=a_{n \sigma^{\prime} k^{\prime}} e^{i p_{\sigma^{\prime} k^{\prime}}(x-n)}+b_{n \sigma^{\prime} k^{\prime}} e^{-i p_{\sigma^{\prime} k^{\prime}}(x-n)}
\end{gathered}
$$

Analogous expansion holds for $x_{n}<x<x_{n+1}$. The matching conditions at $x=n$ read,

$$
\begin{aligned}
& \Psi\left(x=n^{-} ;\left\{S_{n}\right\}\right)=\Psi\left(x=n^{+} ;\left\{S_{n}\right\}\right) \\
& \quad \Psi^{\prime}\left(x=n^{+} ;\left\{S_{n}\right\}\right)-\Psi^{\prime}\left(x=n^{-} ;\left\{S_{n}\right\}\right) \\
& =g \mathbf{s} \cdot \mathbf{S}_{n} \Psi\left(x=n^{-} ;\left\{S_{n}\right\}\right) .
\end{aligned}
$$

Employing the expansion (5) and applying on the left the bra $<k \mid \otimes \chi_{\sigma}^{\dagger}$, the relations (7.,8) imply $4(N+1)$ equations from which the coefficients $a_{n+1 \sigma k}, b_{n+1 \sigma k}$ are expressible in terms of $a_{n \sigma k}, b_{n \sigma k}$. At the end of this procedure (which is briefly detailed here), it yields the transfer matrix $\tau_{n}$ that carries the system from $n-0$ to $n+0$. To be definite, the order of coefficients (which determines the structure of transfer matrices) is $\left(\mathbf{a}_{n \uparrow}, \mathbf{a}_{n \downarrow}, \mathbf{b}_{n \uparrow}, \mathbf{b}_{n \downarrow}\right)$. The required manipulation is to evaluate matrix elements of four $(N+1) \times(N+1)$ matrices $I_{\sigma^{\prime} \sigma}$ defined as operators in spin wave space,

$$
<k^{\prime}\left|I_{\sigma^{\prime} \sigma}\right| k>\equiv<k^{\prime}\left|\otimes \chi_{\sigma^{\prime}}^{\dagger} \mathbf{s} \cdot \mathbf{S}_{n} \chi_{\sigma} \otimes\right| k>
$$

Straightforward calculations yield, (separating $k, k^{\prime}=0$ and $\left.k, k^{\prime} \neq 0\right)$

$$
\begin{aligned}
& <k^{\prime}\left|I_{\uparrow \uparrow}\right| k>=\left[\begin{array}{cc}
\frac{1}{4} & 0 \\
0 & \frac{\delta_{k k^{\prime}}}{4}-\frac{e^{i\left(k-k^{\prime}\right) n}}{2 N}
\end{array}\right], \\
& <k^{\prime}\left|I_{\uparrow \downarrow}\right| k>=\left[\begin{array}{cc}
0 & 0 \\
\frac{e^{-i k^{\prime} n}}{2 \sqrt{N}} & 0
\end{array}\right], \\
& I_{\downarrow \uparrow}=I_{\uparrow \downarrow}^{\dagger}, \\
& I_{\downarrow \downarrow}=-I_{\uparrow \uparrow .} .
\end{aligned}
$$

The transfer matrix across $x=n\left(\tau_{n}\right)$ can now be written down, remembering that in many channel problems, the plane waves are normalized to have unit velocity. In terms of the $4(N+1) \times 4(N+1)$ diagonal matrix,

$$
p^{-\frac{1}{2}}=\operatorname{diag}\left(\mathbf{p}_{\uparrow}^{-\frac{1}{2}}, \mathbf{p}_{\downarrow}^{-\frac{1}{2}}, \mathbf{p}_{\uparrow}^{-\frac{1}{2}}, \mathbf{p}_{\downarrow}^{-\frac{1}{2}}\right),
$$

where $\mathbf{p}_{\sigma}=\left(p_{\sigma 0}, p_{\sigma 1}, . . p_{\sigma N}\right)$ is the vector of $N+1$ channel momenta, the result is,

$$
\begin{aligned}
& \tau_{n}=1+ \\
& \frac{g}{2 i} p^{-\frac{1}{2}}\left[\begin{array}{cccc}
I_{\uparrow \uparrow} & I_{\uparrow \downarrow} & I_{\uparrow \uparrow} & I_{\uparrow \downarrow} \\
I_{\downarrow \uparrow} & I_{\downarrow \downarrow} & I_{\downarrow \uparrow} & I_{\downarrow \downarrow} \\
-I_{\uparrow \uparrow} & -I_{\uparrow \downarrow} & -I_{\uparrow \uparrow} & -I_{\uparrow \downarrow} \\
-I_{\downarrow \uparrow} & -I_{\downarrow \downarrow} & -I_{\downarrow \uparrow} & -I_{\downarrow \downarrow}
\end{array}\right] p^{-\frac{1}{2}} .
\end{aligned}
$$

Propagation between spins is controlled by the diagonal $4(N+1) \times 4(N+1)$ matrix of phases,

$$
\Lambda=\operatorname{diag}\left(e^{i \mathbf{p}_{\uparrow}}, e^{i \mathbf{p}_{\downarrow}}, e^{-i \mathbf{p}_{\uparrow}}, e^{-i \mathbf{p}_{\downarrow}}\right) .
$$

The transfer matrix $T_{n}$ across a unit cell (from $n-1+0$ to $n+0)$ and the total transfer matrix $T$ are then, $T_{n}=\Lambda \tau_{n}$ and $T=\prod_{n=1}^{N} T_{n}$. With some modifications required in case there are evanescent modes, it is not difficult to show that in the above 4 block partition of transfer matrices into $\mathbf{a}$ and $\mathbf{b}$ sectors, the transfer matrices $\tau_{n}, T_{n}$ and $T$ satisfy the current conservation constraint, $\tau_{n} \sigma_{z} \tau_{n}^{\dagger}=\sigma_{z}$. Finally, the matrix of amplitudes $t_{\sigma^{\prime} \sigma}$ with elements $t_{\sigma^{\prime} k^{\prime} \sigma k}(E)$ is simply given by $T_{\mathbf{a a}}^{-1}$.

Conductance and spin transfer coefficients: An experimentally relevant question is the following: Applying a small voltage $V$ across the wire between 0 and $N+1$ and letting a unit flux of electrons at Fermi energy $\varepsilon_{F}$ and spin component $\sigma$ reach it from the left, what is the current $I_{\sigma^{\prime} \sigma}$ of electrons with spin $\sigma^{\prime}$ in the system? The ratio $G_{\sigma^{\prime} \sigma}\left(\varepsilon_{F}\right) \equiv I_{\sigma^{\prime} \sigma} / V$ is the corresponding STC. The conductance per spin is $G_{\sigma}=\sum_{\sigma^{\prime}} G_{\sigma^{\prime} \sigma}$ and the conductance is $G=\sum_{\sigma} G_{\sigma}$. At zero temperature (and for infinitesimal voltage drop $V$ ) the system is in its ground state and only elastic scattering (of the electrons) is allowed. Due to spin conservation, processes involving spin flip are inelastic and hence are forbidden. Thus, the quantities to be calculated at zero temperature and infinitesimal voltage difference are,

$$
G_{\sigma \sigma}\left(\varepsilon_{F}\right)=\left|t_{\sigma 0 \sigma 0}\left(\varepsilon_{F}+E_{0}\right)\right|^{2},
$$

where $\varepsilon_{F}+E_{0}=E$ is the total energy of the system. Evidently, $G_{\uparrow \uparrow}$ is trivial since it involves only the ground state $\mid 0>$ of the spin system. Coupling terms to OMSW, $<k \neq 0\left|\otimes \chi_{\sigma^{\prime}}^{\dagger} \mathbf{s} \cdot \mathbf{S}_{n} \chi_{\uparrow} \otimes\right| 0>=0$ since the initial state $\chi_{\uparrow} \otimes \mid 0>$ is stretched with total spin $N / 2+1 / 2$ while the final state is not. Therefore, the quantum spins are replaced by their averages $<0\left|\mathbf{S}_{n}\right| 0>=S_{n z}$ and spin up incoming electrons are scattered by a sequence of $N$ identical delta function potentials of strength $g / 4$. On the other hand, $G_{\downarrow \downarrow}$ involves virtual OMSW excitations and hence it is non trivial. For examples, it gets second order contributions $<0\left|\otimes \chi_{\downarrow}^{\dagger} \mathbf{s} \cdot \mathbf{S}_{m} \chi_{\uparrow} \otimes\right| k><k\left|\otimes \chi_{\uparrow}^{\dagger} \mathbf{s} \cdot \mathbf{S}_{n} \chi_{\downarrow} \otimes\right| 0>$.

Of special interest are spin flip processes. If the potential drop $e V$ exceeds the gap between the ground state energy $E_{0}$ and the lowest OMSW energy (that is, 
$\mathrm{eV} \geq 2 S E_{Z}$ ), then the process $\uparrow \leftarrow \downarrow$ is energetically feasible even at $T=0$. The electron stays at the Fermi energy and the spin system is heated from $E_{0}$ to $E_{1}$, the energy being supplied from the external voltage. Assuming that linear response is still valid, the corresponding STC is $G_{\uparrow \downarrow}\left(\varepsilon_{F}\right)=\left|t_{\uparrow 1 \downarrow 0}\left(\varepsilon_{F}+E_{0}\right)\right|^{2}$. After the system is excited from the ground state to the lowest OMSW state, the process $\uparrow \leftarrow \downarrow$ is blocked. It can occur again only if the spin system returns to its ground state. This can happen for example by the process $\downarrow \leftarrow \uparrow$ (which, by spin conservation, is allowed if the spin system is in a OMSW state but is otherwise forbidden on the ground state). Finally, consider the spin-flip process encoded in $G_{\downarrow \uparrow}\left(\varepsilon_{F}\right)=\left|t_{\downarrow 0 \uparrow k}\left(\varepsilon_{F}+E_{k}\right)\right|^{2}$. This can happen only at $T>0$ where the spin system contains also a OMSW component. At the end, the spin system cools down to the ground state, but regains the excited component energy from the heat bath.

Possible experimental realization: Out of the four parameters $g, J, \mathbf{H}$ and $\varepsilon_{F}$, the last two are the ones which are easiest to handle and control. However, the strength of the magnetic field is somewhat constrained by the condition $E_{Z}>J S$ justifying projection on the OMSW subspace. The $s-d$ coupling $g$ is material dependent and can have either sign but in general $|g| \gg|J|$. The atomic and electronic Zeeman energies are similar in magnitude, $E_{Z} \sim h_{e}$. Thus, one should concieve a material consisting of itinerant electrons and localized magnetic moments in which the Fermi energy can be tuned by a sutable gate. Possible candidates are magnetic semiconductors (e.g. FeSi) or strongly correlated semimetals such as $C e P$ and $C e S b$.

Results: Calculations are performed on a chain of $N=12$ spins, using $J S=0.06$, and taking $g= \pm 1$ for the electron-atom $s-d$ exchange strength. The atomic and electronic Zeeman energies are taken to be $E_{Z}=h_{e}=0.2$. The Fermi energy is taken with reference to the ground state of the spin system in units of $|J| S$. The results at $T=0$ are presented in figures 1, 2, and 3 below.

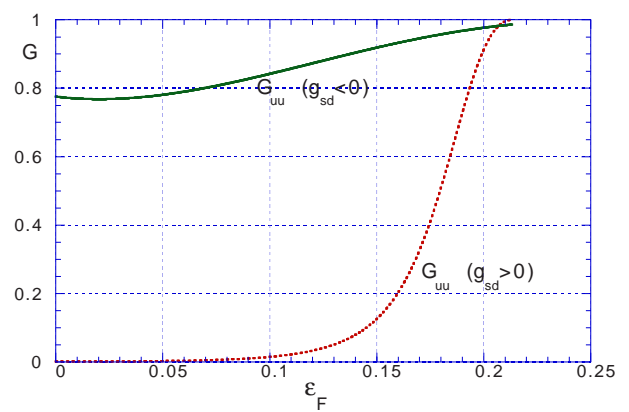

FIG. 1. Non-flip spin transfer coefficient $G_{\uparrow \uparrow}\left(\varepsilon_{F}\right)$ (equation 14) for electron transmission through a chain consisting of $N=12$ spins prepared at the ground state $\mid 0>$. Solid (dashed) lines correspond to $g=-1$, a ferromagnetic $(g=1$, an antiferromagnetic) $s-d$ exchange coupling. Other parameters are $J=0.12, E_{Z}=h_{e}=0.2$.

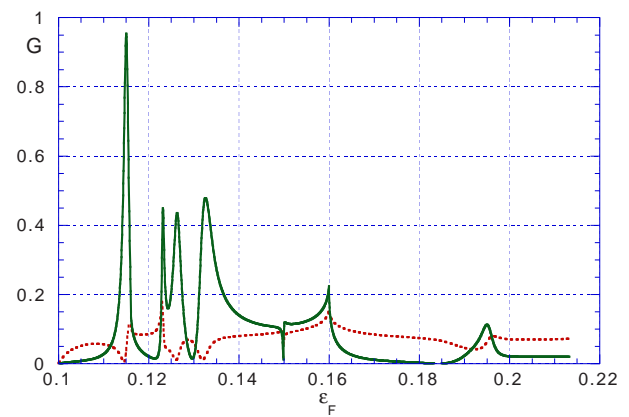

FIG. 2. Non-flip spin transfer coefficient $G_{\downarrow \downarrow}\left(\varepsilon_{F}\right)$ (solid line) and spin flip transfer coefficient $G_{\uparrow \downarrow}\left(\varepsilon_{F}\right)$ (dashed line) for a ferromagnetic $s-d$ exchange coupling $g=-1$. Other specifications are as in figure 1.

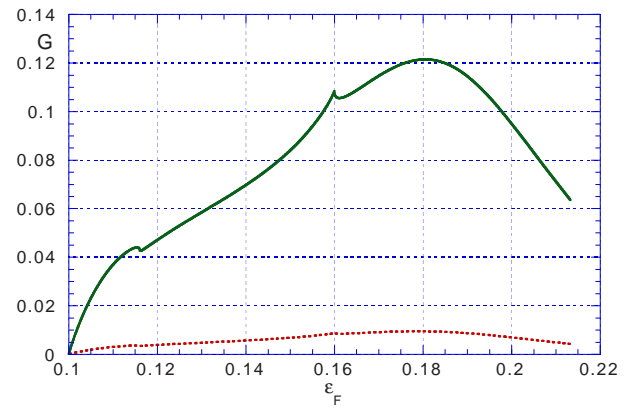

FIG. 3. Same as figure for $g=1$ (an anti ferromagnetic $s-d$ exchange coupling).

As asserted above, the behavior of $G_{\uparrow \uparrow}$ (figure 1 1 ) is somewhat expected since only the $s_{z} S_{n z}$ interaction is effective. For a ferromagnetic $s-d$ coupling the electron encounters a periodic potential composed of attractive $\delta$ functions while for an antiferromagnetic exchange the potentials are repulsive. This leads to a band structure determined by, $\left|\cos k_{0}+\frac{g}{k_{0}} \sin k_{0}\right| \leq 1$, where $k_{0}=\sqrt{\varepsilon_{F}}$. It is easy to see that the above range of $\varepsilon_{F}$ is inside the band.

The patterns of the STC $G_{\downarrow \downarrow}$ and $G_{\uparrow \downarrow}$ (figures 2 for $g=-1$ and 3 for $g=1$ ) have a much richer content. In particular, for a ferromagnetic exchange coupling (figure 21) they display a series of very narrow resonances. It is quite remarkable that in a magnetic field and with ferromagnetic $s-d$ coupling, still, $G_{\downarrow \downarrow}$ approaches unity at resonance. These resonances are novel, that is, they are not the usual ones encountered in resonance tunneling, since there is neither potential scattering here nor double barrier. As discussed above, the pertinent processes involve virtual excitations of the OMSW, and hence, it 
reflects the structure of the electron spin chain system. Unlike potential scattering in which the scattering is effected upon static (quenched) impurities, we encounter here scattering from a quantum system with an internal structure and many non-degenerate levels (the case of degenerate levels is exemplified by the Kondo effect). It is also noticed that these resonances appear only for a ferromagnetic $s-d$ coupling $g<0$. Some light can be shed on these points by inspecting the corresponding bound state problem for a closed system. Namely, the one dimensional wire is closed into a ring of length $N$ and the bound state energies (pertaining to the total system, electron and spin chain) can be computed by solving the equation $\operatorname{det}[T(E)-1]=0$ (in order to avoid orbital effects, the magnetic field is tuned to yield an nteger number of flux quanta through the ring). While there is no simple relation between an open wire conductance rsonances and bound states on a closed wire, it is nevertheless instructive to notice that the bound state energies in the ferromagnetic case are lower than those in the antiferromagnetic case. In fact, the latters fall mostly outside the range of Fermi energies examined in these simulations.

At finite temperature, the spin system can be activated to the lowest OMSW (which, unlike higher OMSW, is not degenerate). The process $\downarrow 1 \leftarrow \uparrow 0$ is then feasible and the relevant STC $G_{\downarrow \uparrow}$ is depicted in figure 1 .

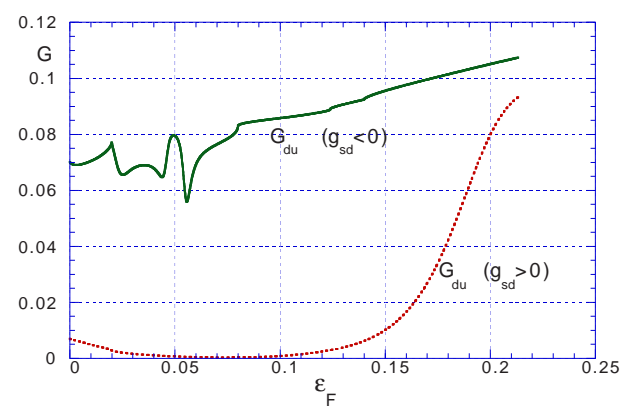

FIG. 4. The spin transfer coefficient $G_{\downarrow \uparrow}\left(\varepsilon_{F}\right)$ for a spin system prepared at the first OMSW state. The value of the $s-d$ exchange constant is $g=\mp 1$ (solid and dashed lines). Other parameters are as in figure 1 .

It mainly reflects the coupling between the ground and lowest OMSW state of the spin system. Higher OMSW are virtually excited in higher orders through non-flip processes, e.g. $(\downarrow 0) \leftarrow(\uparrow k \geq 1) \leftarrow(\uparrow 1)$. The resonant pattern prevails also in this case. Note that the actual value of the STC should be reduced by the activation factor $e^{-\beta\left(E_{1}-E_{0}\right)}$.

Conclusions: The formalism for studying electron transport in a quantum spin chain is developed. Application of an external magnetic field removes the Kondo problem as it lifts the degeneracy of spin states. (For infinite chains it also restores long range order which is otherwise absent due to the Mermin - Wagner theorem). Spin transfer coefficients $G_{\sigma^{\prime} \sigma}$ are evaluated and analyzed as a function of the Fermi energy. Non-flip processes $\uparrow \leftarrow \uparrow$ and $\downarrow \leftarrow \downarrow$ are elastic. The structure of $G_{\uparrow \uparrow}$ is trivial since the problem is equivalent to the scattering of polarized electrons on a sequence of delta function potentials. On the other hand, the behavior of $G_{\downarrow \downarrow}$ is much richer. For a ferromagnetic $s-d$ exchange coupling it displays a novel resonant pattern which is distinct from the one encountered in potential or double barrier transmission. That is, it reflects the complex structure of the electron spin-chain system. Spin-flip processes are inelastic. The transition $\uparrow \leftarrow \downarrow$ is endothermic, and is feasible at $T=0$ if the potential difference across the spin system overcomes the energy gap between the ground and lowest OMSW states. The transition $\downarrow \leftarrow \uparrow$ is exothermic and feasible at $T>0$ if OMSW are activated. Although spin flip coefficients are small relative to non spin flip ones, they are still sizable. In passing, we note that there are interesting theoretical works discussing the pertinent many body problem [10] 11].

Acknowledgements: We thank M. Yamanaka, T. Koma, E. Kogan and K. Kikoin for helpful comments. Y. A. acknowledges supports by DIP German Israel Cooperation, Israeli SF grants Center of Excellence, Many Body Effects in Resonance Tunneling and US-Israel BSF Dynamical Instabilities in quantum dots. Y.T. acknowledges support from the NEDO joint research program (NTDP-98).

[1] D. Weis, Physics World 13,23 (2000); R. Fiederling et al. Nature, 402, 787 (1999); Y. Ohno et al. Nature, 402, 790 (1999); A. T. Filip, B. H. Hoving, F. J. Jedema, and B. J. van Wees, Phys. Rev. B62,9996 (2000).

[2] W. G. van der Wiel et al. , Science, 289, 2105 (2000).

[3] P. Bruno, Phys. Rev. Lett., 83, 2425 (1999).

[4] N. García et al., Phys. Rev. Lett., 82, 2923 (1999).

[5] H. Oshima and K. Miyano, Appl. Phys. Lett. 73, 2203 (1998).

[6] T. Ono et al., Appl. Phys. Lett. 75, 1622 (1999).

[7] H. Imamura, N. Kobayashi, S. Takahashi and S. Maekawa, Phys. Rev. Lett., 84, 1003 (2000).

[8] M. Yamanaka and T. Koma, J. Magnetical Society of Japan 23, 141 (1999).

[9] G. Tatara and Y. Tokura, Solid State Communication, 116, 533 (2000).

[10] J. J. Betouras and S. Fujimoto, Phys. Rev. B59, 529 (1999).

[11] O. Zachar, cond-mat/0011312. 